\title{
Eight-year follow-up of the Clopidogrel After Surgery for Coronary Artery Disease (CASCADE) trial
}

\author{
Ali Hage, MD, ${ }^{a}$ Pierre Voisine, MD, ${ }^{\mathrm{a}}$ Fernanda Erthal, MD, ${ }^{\mathrm{b}}$ Éric Larose, MD, ${ }^{\mathrm{c}}$ David Glineur, MD, PhD, ${ }^{\mathrm{d}}$ \\ Benjamin Chow, MD, ${ }^{\mathrm{b}}$ Hugo Tremblay, RN, ${ }^{\mathrm{a}}$ Jacqueline Fortier, MSc, ${ }^{\mathrm{d}}$ Gifferd Ko, MSc, ${ }^{\mathrm{d}}$ Dai Une, MD, \\ Michael Farkouh, MD, MS, ${ }^{\mathrm{f}}$ Thierry G. Mesana, MD, PhD, ${ }^{\mathrm{d}}$ Michel LeMay, MD, ${ }^{\mathrm{b}}$
} Alexander Kulik, MD, MPH, ${ }^{\mathrm{g}}$ and Marc Ruel, MD, $\mathrm{MPH}^{\mathrm{d}}$

\section{ABSTRACT}

Objective: In this 8 years' follow-up study, we evaluated the long-term outcomes of the addition of clopidogrel to aspirin during the first year after coronary artery bypass grafting, versus aspirin plus placebo, with respect to survival, major adverse cardiac, or major cerebrovascular events, including revascularization, functional status, graft patency, and native coronary artery disease progression.

Methods: In the initial Clopidogrel After Surgery for Coronary Artery Disease trial, 113 patients were randomized to receive either daily clopidogrel $(n=56)$ or placebo $(\mathrm{n}=57)$, in addition to aspirin, in a double-blind fashion for 1 year after coronary artery bypass grafting. All patients were re-evaluated to collect long-term clinical data. Surviving patients with a glomerular filtration rate $>30 \mathrm{~mL} / \mathrm{min}$ were asked to undergo a coronary computed tomography angiogram to evaluate the late saphenous vein graft patency and native coronary artery disease progression.

Results: At a median follow-up of 7.6 years, survival rate was $85.5 \% \pm 3.8 \%$ ( $P=.23$ between the 2 groups). A trend toward enhanced freedom from all-cause death or major adverse cardiac or cerebrovascular events, including revascularization, was observed in the aspirin-clopidogrel group $(P=.11)$. No difference in functional status or freedom from angina was observed between the 2 groups $(P>.57)$. The long-term patency of saphenous vein graft was $89.11 \%$ in the aspirin-clopidogrel group versus $91.23 \%$ in the aspirin-placebo group $(P=.79)$. A lower incidence of moderate to severe native disease progression was observed in the aspirin-clopidogrel group versus the aspirin-placebo group ( 7 out of 122 vs 13 out of 78 coronary segments that showed progression, respectively [odds ratio, $0.3 \pm 0.2 ; 95 \%$ confidence interval, $0.1-0.8 ; P=.02$ ]).

Conclusions: At 8 years' follow-up, the addition of clopidogrel to aspirin during the first year after coronary artery bypass grafting exhibited a lower incidence of moderate to severe progression of native coronary artery disease and a trend toward higher freedom from major adverse cardiac or cerebrovascular

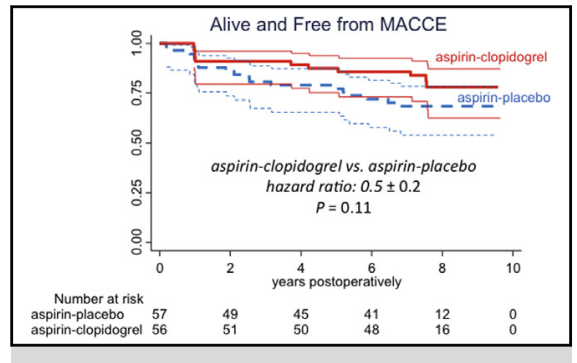

A trend toward long-term freedom from all-cause death or MACCE/R was seen with 1 year of DAPT.

\section{Central Message}

The addition of clopidogrel to aspirin during the first year after CABG correlates with a higher freedom from MACCE/R or death and a lower incidence of moderate to severe progression of native CAD.

\section{Perspective}

This follow-up study is the first of its kind to evaluate the long-term effect of dual antiplatelet therapy on clinical functional status and on both graft patency and native coronary artery disease progression 8 years after coronary artery bypass grafting. With a $100 \%$ clinical follow-up rate, this study provides very high-quality, long-term, contemporary, clinical trial-based information regarding clinical and subclinical outcomes of coronary artery bypass grafting.

See Editorial Commentary page 223. events, including revascularization, or death in the aspirin-clopidogrel group.

Clinical Trial Registration: http://www.clinicaltrials.gov. Unique identifier: NCT00228423. (J Thorac Cardiovasc Surg 2018;155:212-22)

\footnotetext{
From the Divisions of a Cardiac Surgery and ${ }^{\mathrm{c}}$ Cardiology, Québec Heart and Lung Institute, Québec City, Québec; Divisions of ${ }^{b}$ Cardiology and ${ }^{\mathrm{d}}$ Cardiac Surgery, University of Ottawa Heart Institute, Ottawa, Ontario, Canada; ${ }^{e}$ the Division of Cardiovascular Surgery, Yamato Seiwa Hospital, Yamato, Japan; ${ }^{\mathrm{f}}$ the Division of Cardiology, Mount Sinai Hospital, New York, NY; and ${ }^{\mathrm{g}}$ the Division of Cardiovascular Surgery, Lynn Heart \& Vascular Institute, Boca Raton, Fla.

M.R. has received research support from the Bristol-Myers Squibb Sanofi Canada Partnership for the conduct of the Clopidogrel after Surgery for Coronary Artery Disease trial. M.R. and A.K. have received funds from the Physician Services Incorporated Foundation. B.C. receives research support from GE Healthcare and educational support from TeraRecon Inc.
}

Read at the 96th Annual Meeting of the American Association for Thoracic Surgery, Baltimore, Maryland, May 14-18, 2016.

Received for publication May 30, 2016; revisions received May 18, 2017; accepted for publication June 14, 2017; available ahead of print July 20, 2017.

Address for reprints: Marc Ruel, MD, MPH, Division of Cardiac Surgery, University of Ottawa Heart Institute, 40 Ruskin St, Ste 3402, Ottawa, Ontario, Canada (E-mail: mruel@ottawaheart.ca).

$0022-5223 / \$ 36.00$

Copyright (C) 2017 by The American Association for Thoracic Surgery

http://dx.doi.org/10.1016/j.jtcvs.2017.06.039 


\section{Abbreviations and Acronyms \\ BITA = bilateral internal thoracic arteries \\ CABG = coronary artery bypass grafting \\ CAD = coronary artery disease \\ CASCADE $=$ Clopidogrel After Surgery for Coronary Artery Disease \\ CCTA $=$ coronary computed tomographic angiography \\ CRF = case report form \\ DAPT $=$ dual antiplatelet therapy \\ ITA $=$ internal thoracic artery \\ MACCE = major adverse cardiac or cerebrovascular events \\ $\mathrm{MACCE} / \mathrm{R}=$ major adverse cardiac or cerebrovascular events including revascularization \\ SVG = saphenous vein graft}

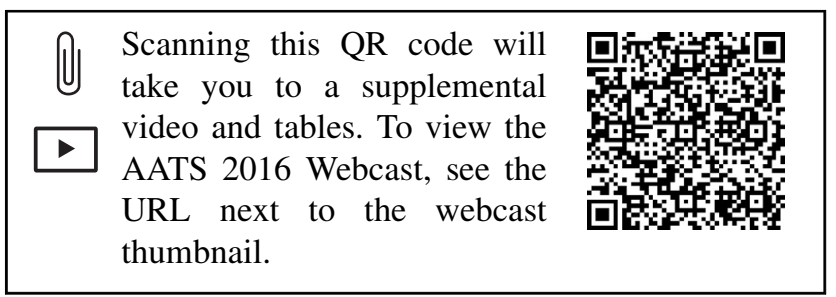

Coronary artery bypass grafting (CABG) using saphenous vein grafts (SVGs) and 1 or both internal thoracic arteries (ITAs) has long been used as a treatment for coronary artery disease (CAD). Following CABG, native CAD continues to progress and constitutes an important contributor to subsequent major adverse cardiac events. ${ }^{1}$ On the other hand, SVGs also undergo a process of conduit attrition composed of 3 interlinked stages: early, often nonocclusive, thrombosis then intimal hyperplasia, which sets the ground for the development of later atherosclerosis and eventual occlusion. $^{2}$ Aspirin has been shown to be effective against early thrombosis; however, in animal models, only clopidogrel was shown to prevent intimal hyperplasia. ${ }^{3,4}$ As a result of CAD progression and SVG disease, patients who have undergone $\mathrm{CABG}$ are susceptible to late ischemic heart events. ${ }^{1,2,5}$

To address this problem, our Clopidogrel After Surgery for Coronary Artery Disease (CASCADE) randomized controlled trial (ClinicalTrials.gov identifier: NCT00228423) examined the effect of dual antiplatelet therapy (DAPT), combining aspirin and clopidogrel (vs placebo), on SVG intimal hyperplasia, graft patency, and progression of CAD after CABG. CASCADE demonstrated that compared with aspirin monotherapy, the combination of aspirin plus clopidogrel did not significantly reduce the process of SVG intimal hyperplasia at 1 year after CABG. ${ }^{6}$ However, DAPT was associated with a slowing of the progression of native CAD after surgery. ${ }^{7}$ Notably, other trials, such as a trial that looked at 12 or 30 months of DAPT after drug-eluting stents, ${ }^{8}$ recently indicated that combining aspirin and a thienopyridine, for 30 months after drug-eluting coronary stents, was associated with a reduction in the incidence of major adverse cardiac or cerebrovascular events (MACCE) beyond that attributable to stent complications, likely as a result of less native CAD progression.

In the present follow-up study of the CASCADE trial, conducted 8 years postrandomization, we evaluated long-term outcomes in the 2 treatment groups. Patients who received aspirin-clopidogrel or aspirin-placebo for 1 year after CABG were followed 7 years later with respect to survival; MACCE, including revascularization (MACCE/R); functional status; graft patency; and native CAD progression. Our hypothesis was that treatment with DAPT during the initial post-CABG year could influence the trajectory of subsequent events, despite intercurrent antiplatelet therapy or not. The intervention was 1 year of DAPT versus aspirin alone, and it was randomized and double blind. The analysis undertaken 7 years later constitutes an open-label, long-observational follow-up phase. Such follow-up would allow for a unique opportunity to ascertain the late results of the CASCADE trial intervention with respect to long-term clinical and functional data, graft, and native $\mathrm{CAD}^{2}$ and a prospective assessment of the late results of $\mathrm{CABG}$ within the monitored context of a randomized controlled trial cohort.

\section{METHODS}

This study was a long-term follow-up at 8 years of the CASCADE trial, which was a dual-center (University of Ottawa Heart Institute, Ottawa, Ontario, Canada, and Hôpital Laval, Québec City, Québec, Canada) randomized placebo-controlled trial designed to primarily investigate the effect of DAPT on the development of SVG disease 1 year after CABG. ${ }^{6}$

The objective of this study is to evaluate the long-term outcomes in the 2 treatment groups. Patients who had received aspirin-clopidogrel or aspirin-placebo for 1 year after CABG, were followed 8 years postoperatively with respect to survival, MACCE/R, functional status, graft patency, and native CAD progression. Each of these end points was considered independently and the end points were not combined into a single composite time-related event. Our definition of MACCE and MACCE/R did include myocardial infarction, stroke, cardiovascular death, as well as the same components plus the addition of myocardial revascularization, respectively. We separated MACCE/R from all-cause death to evaluate overall survival (freedom from all-cause death) as a separate end point.

Specific institutional review board review and authorization was obtained at both centers to complete this CASCADE follow-up study. A new protocol, including functional status determinations and coronary 
computed tomographic angiography (CCTA), was independently reviewed and approved by each institutional research ethics board, and informed patient consent was obtained for each patient before either completing the detailed questionnaire and the CCTA.

\section{Population}

In the initial CASCADE trial, 113 patients who underwent elective CABG with $\geq 2$ SVGs were randomized to receive either daily clopidogrel $75 \mathrm{mg}(\mathrm{n}=56)$ or placebo $(\mathrm{n}=57)$, in addition to aspirin $162 \mathrm{mg}$ daily, for 1 year after CABG. The treatment strategy was double-blind, randomized, and placebo-controlled, initiated right after the operation, and continued during the first year of the trial. After the first year of the trial, the choice of antiplatelet regimen was left to the patient's physician. This follow-up study was not an a priori planned long-term analysis of the CASCADE trial and the study number of 113 is the sample size that was determined during the initial phase of the CASCADE trial. Statistical power at that time was consistent with the surrogate end point of intravascular ultrasound determined graft intimal area. ${ }^{6}$

In the original study, a preoperative coronary angiogram had been performed in all 113 patients, and a postoperative coronary angiogram 1 year after CABG was performed in 92 eligible patients at the 2 centers ( $\mathrm{n}=46$ for both treatment groups).

\section{Patient Follow-up}

A follow-up Case Report Form (CRF) was designed to collect long-term clinical (cardiac mortality, myocardial infarction, rehospitalization for coronary ischemia or coronary intervention, stroke, and bleeding), and functional data for each patient. The CRF also included a Seattle Angina Questionnaire, with each of its 5 subcomponents, to assess the long-term functional aspects of CAD.

An attempt was made to contact all 113 patients from the initial CASCADE trial. Packages including an introduction letter and a copy of the consent form were sent to addresses retrieved from the CASCADE database. Two weeks later, patients were contacted by telephone and the study was explained. Patients were then asked, according to their availability, to either give a verbal consent and to complete the CRF by telephone, or to come to the hospital to complete the CRF.

\section{CCTA}

Surviving patients with a glomerular filtration rate $>30 \mathrm{~mL} / \mathrm{min}$ were asked to undergo a CCTA to evaluate the long-term effects of 1 year of DAPT versus aspirin monotherapy on late graft patency and native CAD progression.

CCTAs of native coronary arteries and grafts were analyzed in blinded fashion to treatment by trained imaging cardiologists.

For the native coronary arteries, the CCTA coronary stenosis results were reported using a 4-grade system where each native coronary artery segment was graded based on its most severe stenosis, regardless of the localization of the stenosis within the segment. Arterial segments were classified as proximal, middle, and distal. Patency data obtained preoperatively and 1-year post CABG were retrospectively regraded using this system, which was defined as grade $0=$ normal, grade $1=$ mild $(<50 \%)$, grade $2=$ moderate $(50 \%-69 \%)$, grade $3=$ severe $(\geq 70 \%)$, and grade $4=$ occluded. Then, based on the difference between the grading preoperatively and at the time of follow-up, native CAD progression was graded as 0 (no progression), mild progression (1 grade of worsening), moderate ( 2 grades of worsening), severe ( 3 grades of worsening), or complete (4 grade of worsening). When a coronary artery segment was subsequently stented, we a priori made the progression a grade 3 if the stented artery was $<70 \%$ stenosis at latest evaluation, and grade 4 if the stented artery had $70 \%$ or more stenosis within the stent.

A competing risks analysis was used to estimate late graft occlusion in both treatment groups. In this analysis, patients who died from a cardiac cause or who experienced MACCE/R and did not undergo late CCTA (either due to refusal or death from a noncardiac cause), were considered as having all grafts occluded. ${ }^{9}$

\section{Statistical Analysis}

Data were analyzed using Stata version 14.1 (College Station, Tex). Categorical and ordinal data were analyzed with a Fisher exact test. Parametric tests were used for continuous data. Survival was examined by using Kaplan-Meier actuarial methods and log rank tests. The progression of native $\mathrm{CAD}$ was examined by using generalized estimating equations with gamma distribution, accounting for each patient as a cluster in the CAD progression of individual arterial segments.

\section{RESULTS \\ Survival and Clinical Events}

Figure 1 displays the flow chart of the study. Baseline patient characteristics were balanced between the 113 patients, between the aspirin-clopidogrel or aspirinplacebo group, at the inception of the trial. ${ }^{6}$ After the trial's first year, the frequencies of use of clopidogrel and aspirin were similar between the 2 treatment groups $(P>.37)$.

Information about MACCE/R was complete for all patients, with a median clinical follow-up of 7.6 years. For the 14 deceased patients, MACCE/R information was completed with the primary care physician or a first-degree family member to the best of their knowledge. Not all secondary characteristics were collected for each patient because some patients had died or preferred to complete the questionnaire over the telephone due to an inability or unwillingness to come to the hospital to show current medications, be interviewed, and thoroughly complete the CRF with the follow-up team.

Survival of patients at 8 years was $88.5 \% \pm 5.0 \%$ in the aspirin-clopidogrel group and $83.2 \% \pm 5.2 \%$ in the aspirin-placebo group (hazard ratio [HR], $0.5 \pm 0.3 ; 95 \%$ confidence interval $[\mathrm{CI}], 0.2-1.5$; aspirin-clopidogrel vs aspirin-placebo group; $P=.23$ ) (Figure 2). Table 1 shows the patient characteristics in the 99 patients who were alive at follow-up. Surviving patients were significantly older and more often hypertensive in the aspirin-placebo group. The causes of death and MACCE/R are reported in Table 2.

A first MACCE/R occurred in 21 of 113 patients, at a mean of $6.7 \pm 2.5$ years. There was no difference in the incidence of MACCE/R between groups (HR, $0.7 \pm 0.3$; 95\% CI, 0.3-1.7; aspirin-clopidogrel vs aspirin-placebo; $P=.41$ ) (Figure 3).

Taken together, a trend toward enhanced freedom from all-cause death or MACCE/R was observed in the aspirinclopidogrel group versus the aspirin-placebo group (HR, $0.5 \pm 0.2 ; 95 \%$ CI, 0.3-1.1; aspirin-clopidogrel vs aspirin-placebo; $P=.11$ ) (Figure 4).

A robust set of factors between those patients who experienced and those who did not experience MACCE/R was explored. Table E1 shows that patients who experienced a MACCE/R (myocardial infarction, stroke, coronary revascularization, or all-cause death) had similar 


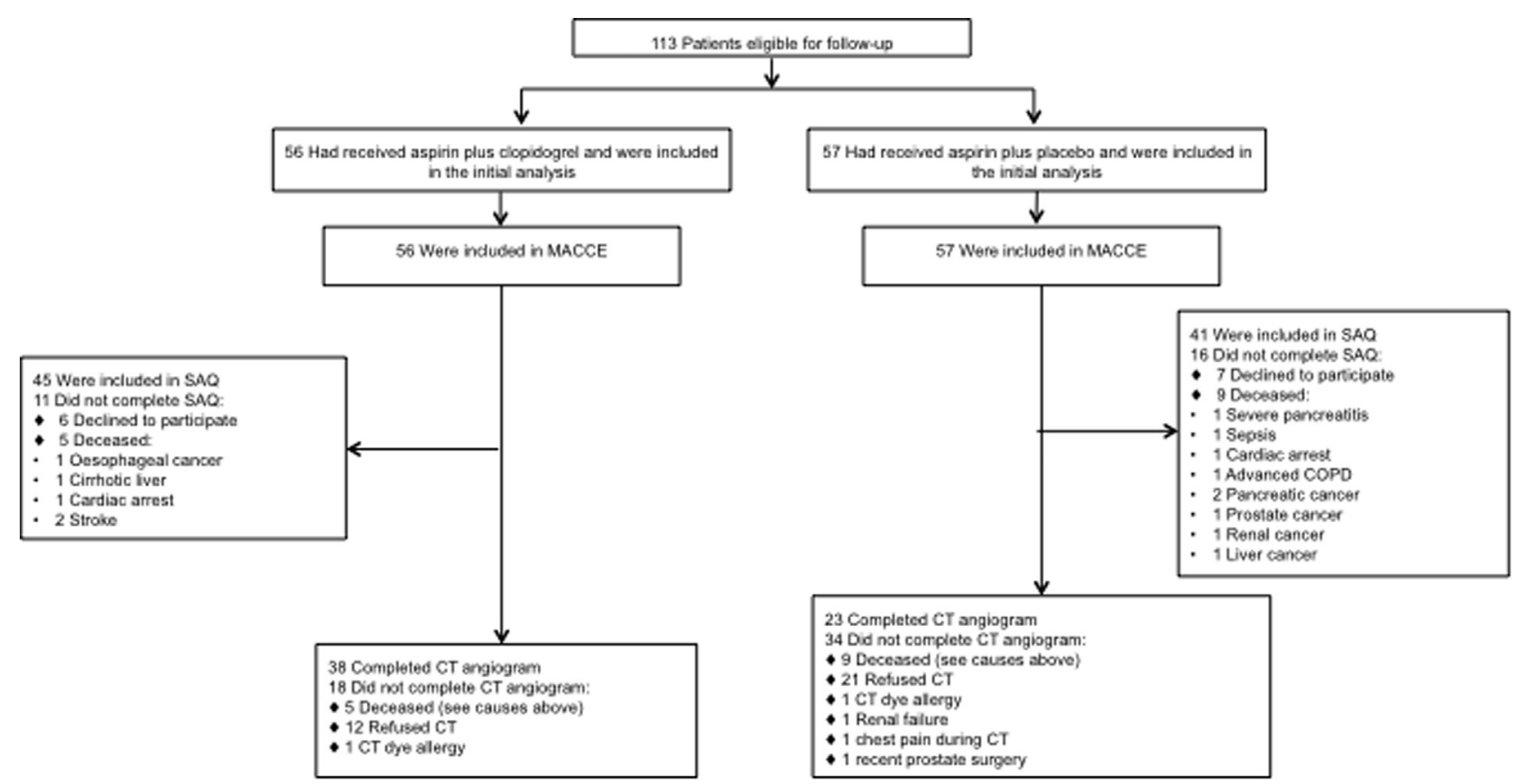

FIGURE 1. Study flow chart. MACCE, Major adverse cardiac or cerebrovascular events; $S A Q$, Seattle Angina Questionnaire; $C O P D$, chronic obstructive pulmonary disease; $C T$, computed tomography.

characteristics between both treatment groups. Table E2 shows that patients who did not experience a MACCE/R also had similar characteristics between both treatment groups, except for age (patients in the aspirin-clopidogrel group were slightly younger than the patients in the aspirin-placebo group).

A sensitivity analysis restricting the data to the 95 patients who received the allocated treatment for the full year of protocol duration revealed an HR for MACCE/R or death of $0.6 \pm 0.2 ; 95 \% \mathrm{CI}, 0.4-1.4 ; P=.27$, aspirin-clopidogrel versus aspirin-placebo group. Another sensitivity analysis focusing solely on the 30 patients who

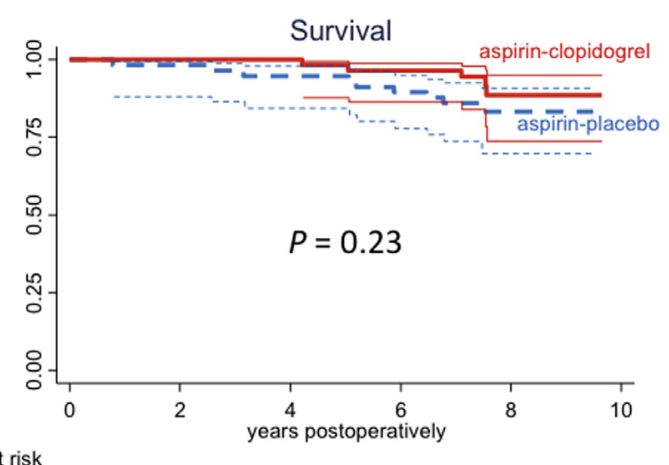

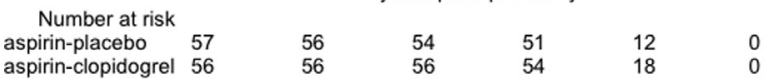

FIGURE 2. Survival of the aspirin-clopidogrel group versus the aspirin-placebo group over the study period. Thick lines (full red lines for the aspirin-clopidogrel group and dashed blue lines for the aspirin-placebo group) depict the point estimates, whereas thin lines represent the $95 \%$ confidence limits. had had an acute coronary syndrome within 6 weeks before the initial randomization did not reveal a trend toward a difference between groups $(P=.74)$.

\section{Functional Status}

Eighty-six of 99 living patients $(86.9 \%)$ answered the detailed functional status questionnaire, with no significant difference between the 2 groups ( 45 of 51 patients in the aspirin-clopidogrel group and 41 of 48 patients in the aspirin-placebo group; $P=.77$ ). Table 3 shows Seattle Angina Questionnaire completion rates and the patients' responses to each of its 5 subcomponents, with very high scores and no significant difference between the 2 groups.

\section{Long-term Fate of CABGs}

Sixty-six of 99 living patients $(67 \%)$ underwent CCTA (39 of 51 patients in the aspirin-clopidogrel group, and 27 of 48 patients in the aspirin-placebo group; $P=.05$ ). However, CCTA could not be completed in 5 patients due to the following reasons: 1 patient with dye allergy in the aspirin-clopidogrel group and 1 with dye allergy, 1 with renal insufficiency, 1 with chest pain during CCTA, and 1 with recent prostate cancer in the aspirin-placebo group. Ultimately, there were significantly more patients in the aspirin-clopidogrel group who completed the CCTA (38 patients in the aspirin-clopidogrel group and 23 patients in the aspirin-placebo group; $P=.008$ ). Baseline characteristics of those who underwent CCTA were explored. Table E3 shows that patients had similar characteristics between both treatment groups. 
TABLE 1. Characteristics of patients at the time of follow-up

\begin{tabular}{|c|c|c|c|}
\hline Characteristic & Aspirin-clopidogrel $(\mathrm{n}=\mathbf{5 1})$ & Aspirin-placebo $(n=48)$ & $P$ value \\
\hline Age $(y)$ & $71.86 \pm 7.73$ & $75.33 \pm 7.64$ & .03 \\
\hline Female $(\%)$ & $7.8(4 / 51)$ & $12.5(6 / 48)$ & .52 \\
\hline Diabetes mellitus (\%) & $33.3(17 / 51)$ & $45.8(22 / 48)$ & .22 \\
\hline Hypertension $(\%)$ & $64.7(33 / 51)$ & $83.3(40 / 48)$ & .04 \\
\hline CCS class 3 or $4(\%)$ & $12.8(6 / 47)$ & $9.8(4 / 41)$ & .75 \\
\hline NYHA III or IV $(\%)$ & $4.3(2 / 47)$ & $4.9(2 / 41)$ & 1.00 \\
\hline \multicolumn{4}{|c|}{ Medication use at the time of follow-up } \\
\hline ACE inhibitor $(\%)$ & $43.5(20 / 46)$ & $48.8(20 / 41)$ & .67 \\
\hline Beta blocker $(\%)$ & $56.5(29 / 46)$ & $68.3(28 / 41)$ & .66 \\
\hline Lipid-lowering $(\%)$ & $91.3(42 / 46)$ & $97.6(40 / 41)$ & .36 \\
\hline Digoxin $(\%)$ & $2.2(1 / 46)$ & $0(0 / 41)$ & 1.00 \\
\hline Amiodarone $(\%)$ & $2.2(1 / 46)$ & $0(0 / 41)$ & 1.00 \\
\hline Calcium channel blocker $(\%)$ & $21.7(10 / 46)$ & $34.2(14 / 41)$ & .23 \\
\hline Oral hypoglycemic $(\%)$ & $32.6(15 / 46)$ & $41.5(17 / 41)$ & .50 \\
\hline Insulin $(\%)$ & $6.5(3 / 46)$ & $2.4(1 / 41)$ & .62 \\
\hline Aspirin $(\%)$ & $87.0(40 / 46)$ & $87.8(36 / 41)$ & 1.00 \\
\hline Clopidogrel (\%) & $10.9(5 / 46)$ & $19.5(8 / 41)$ & .37 \\
\hline Proton pump inhibitor $(\%)$ & $21.7(10 / 46)$ & $41.5(17 / 41)$ & .06 \\
\hline Vitamin $\mathrm{K}$ antagonist (\%) & $6.5(3 / 46)$ & $4.9(2 / 41)$ & 1.00 \\
\hline
\end{tabular}

CCS, Canadian Cardiovascular Society; NYHA, New York Heart Association; $A C E$, angiotensin converting enzyme.

At a mean of $8.0 \pm 0.5$ years, 233 grafts in 61 patients were evaluated by CCTA. The 8-year patency of ITA and SVG was, respectively, $91.49 \%$ and $89.11 \%$ in the aspirin-clopidogrel group versus $92.31 \%$ and $91.23 \%$ in the aspirin-placebo group $(P=1.00$ and $P=.79$, respectively). Table 4 shows the characteristics of the grafts evaluated.

A competing risks analysis was used to estimate late graft occlusion in both treatment groups. In this analysis, patients who died from a cardiac cause or who experienced MACCE/R and did not undergo late CCTA (either due to refusal or death from a noncardiac cause), were considered as having all grafts occluded. ${ }^{9}$ Based on this analysis, 279 grafts were evaluated (166 grafts in the aspirin-clopidogrel group and 113 grafts in the aspirin-placebo group) and the percentage of possible (and likely overestimated) graft occlusion was 18.7\% (31 out of 166) in the aspirin-clopidogrel group, and $32.7 \%$ (37 out of 113) in the aspirin-placebo group (odds ratio, $0.5 \pm 0.2 ; 95 \%$ CI, 0.3-0.8; $P=.01$ ).

\section{Evolution of Native CAD}

Two hundred native coronary disease segments were serially evaluated between preoperative (angiogram), 1 year (coronary angiogram and intravascular ultrasound), and 8-year (CCTA) follow-up. Table 5 shows the distribution of native CAD progression for each group.

Using a generalized estimating equation, the average progression of coronary lesions was $0.23 \pm 0.14$ grades less in the aspirin-clopidogrel group than in the aspirin-placebo group $(P=.10)$. Lesions that had moderate or severe (more than mild) progression were 7 out of 122 $(5.7 \%)$ in the aspirin-clopidogrel group versus 13 out of $78(16.7 \%)$ in the aspirin-placebo group (odds ratio, $0.3 \pm 0.2 ; 95 \% \mathrm{CI}, 0.1-0.8 ; P=.02)$. The latter was driven by the difference between preoperative to 8 -year follow-up because there was no difference in the incidence of more than mild native CAD progression $(1.8 \%$ vs $4.3 \%$ in aspirin-clopidogrel vs aspirin-placebo groups, respectively; $P=.37$ ) between the 1-year angiogram and the 8-year CCTA follow-up between the 2 groups.

\section{Bleeding}

The incidence of major bleeding was $1.8 \%$ in the aspirin-clopidogrel group, and $7.0 \%$ in the aspirinplacebo group $(P=.36)$. The incidence of minor bleeding was $5.4 \%$ in the aspirin-clopidogrel group and $8.8 \%$ in the aspirin-placebo group $(P=.72)$.

\section{DISCUSSION}

This follow-up study is the first of its kind to evaluate the long-term effect of DAPT on clinical functional status and on both graft patency and native CAD progression 8 years after CABG. With a $100 \%$ clinical follow-up rate, this study provides very-high-quality, long-term, contemporary, clinical trial-based information regarding the clinical and subclinical outcomes of CABG.

Our hypothesis was that treatment with DAPT during the initial post-CABG year could influence the incidence of subsequent events, despite intercurrent antiplatelet therapy. 
TABLE 2. Description of major adverse cardiac or cerebrovascular events, including revascularization (MACCE/R) and deaths in the aspirinclopidogrel group and the aspirin-placebo group

\begin{tabular}{lllcc}
\hline & \multicolumn{2}{c}{ Description of the MACCE and death events } \\
\cline { 2 - 5 } Group & $\begin{array}{c}\text { Protocol } \\
\text { completed }\end{array}$ & $\begin{array}{c}\text { Days on } \\
\text { protocol }\end{array}$ & $\begin{array}{c}\text { Type of } \\
\text { MACCE event }\end{array}$ & $\begin{array}{c}\text { Time from the operation } \\
\text { (y)/age at MACCE }\end{array}$ \\
\hline Aspirin-clopidogrel $(\mathbf{n}=\mathbf{1 1})$ & & & \\
\hline Patients alive with MACCE $(\mathrm{n}=7)$ & & & \\
1 & No & 30 & PCI & $0.92 / 62$ \\
2 & Yes & 365 & PCI & $0.97 / 63$ \\
3 & Yes & 365 & PCI & $1.01 / 53$ \\
4 & Yes & 365 & PCI & $1.01 / 61$ \\
5 & Yes & 365 & PCI & $1.07 / 69$ \\
6 & Yes & 365 & PCI & $3.72 / 78$ \\
7 & No & Ischemic stroke & $7.54 / 79$ \\
\hline
\end{tabular}

Timing of the event from the operation $(y) / a g e$ at

MACCE

\begin{tabular}{|c|c|c|c|c|}
\hline \multicolumn{5}{|c|}{ Patients deceased without MACCE $(n=2)$} \\
\hline 1 & Yes & 365 & Cirrhotic liver & $5.05 / 71$ \\
\hline \multirow[t]{2}{*}{2} & Yes & 365 & Esophageal cancer & $7.1 / 83$ \\
\hline & & & $\begin{array}{c}\text { Type of MACCE } \\
\text { event/timing } \\
\text { from the operation } \\
\text { (y)/age at MACCE }\end{array}$ & $\begin{array}{c}\text { Cause of death/timing from } \\
\text { the operation }(y) / a g e\end{array}$ \\
\hline \multicolumn{5}{|c|}{ Patients deceased with history of MACCE $(n=2)$} \\
\hline 1 & Yes & 365 & Cardiac arrest $/ 4.22 / 70$ & Cardiac arrest $/ 4.22 / 70$ \\
\hline 2 & Yes & 365 & Ischemic stroke/7.55/82 & Ischemic stroke/7.55/82 \\
\hline \multicolumn{5}{|c|}{ Aspirin-placebo group $(n=18)$} \\
\hline \multicolumn{5}{|c|}{ Patients alive with MACCE $(\mathrm{n}=9)$} \\
\hline 1 & No & 37 & Ischemic stroke & $0.31 / 69$ \\
\hline 2 & Yes & 365 & PCI & $0.85 / 77$ \\
\hline 3 & Yes & 365 & PCI & $0.98 / 67$ \\
\hline 4 & Yes & 365 & PCI & $0.99 / 71$ \\
\hline 5 & Yes & 365 & PCI & $1.15 / 69$ \\
\hline 6 & Yes & 365 & Ischemic stroke & $1.84 / 62$ \\
\hline 7 & Yes & 365 & PCI & $1.92 / 73$ \\
\hline 8 & Yes & 365 & PCI & $2.18 / 57$ \\
\hline 9 & Yes & 365 & Ischemic stroke & $5.37 / 66$ \\
\hline \multicolumn{5}{|c|}{ Patients deceased without MACCE $(n=6)$} \\
\hline 1 & No & 124 & Renal cancer & $2.55 / 74$ \\
\hline 2 & Yes & 365 & Liver cancer & $3.26 / 75$ \\
\hline 3 & Yes & 365 & Pancreatic cancer & $5.06 / 82$ \\
\hline 4 & Yes & 365 & Prostate cancer & $5.19 / 80$ \\
\hline 5 & Yes & 365 & COPD & $5.88 / 88$ \\
\hline \multirow[t]{2}{*}{6} & Yes & 365 & Pancreatic cancer & $6.47 / 77$ \\
\hline & & & $\begin{array}{c}\text { Type of MACCE } \\
\text { event/timing } \\
\text { from the operation } \\
\text { (y)/age at MACCE }\end{array}$ & $\begin{array}{c}\text { Cause of death/timing from } \\
\text { the operation }(y) / a g e\end{array}$ \\
\hline \multicolumn{5}{|c|}{ Patients deceased with history of MACCE $(n=3)$} \\
\hline 1 & No & 67 & Ischemic stroke/0.19/69 & Sepsis/0.77/69 \\
\hline 2 & Yes & 365 & Myocardial infarction/6.79/70 & Severe pancreatitis/6.79/70 \\
\hline 3 & Yes & 365 & $\mathrm{PCI} / 1.05 / 70$ & Cardiac arrest/7.46/76 \\
\hline
\end{tabular}

$M A C C E$, Major adverse cardiac or cerebrovascular events; $P C I$, percutaneous coronary intervention; $C O P D$, chronic obstructive pulmonary disease. 


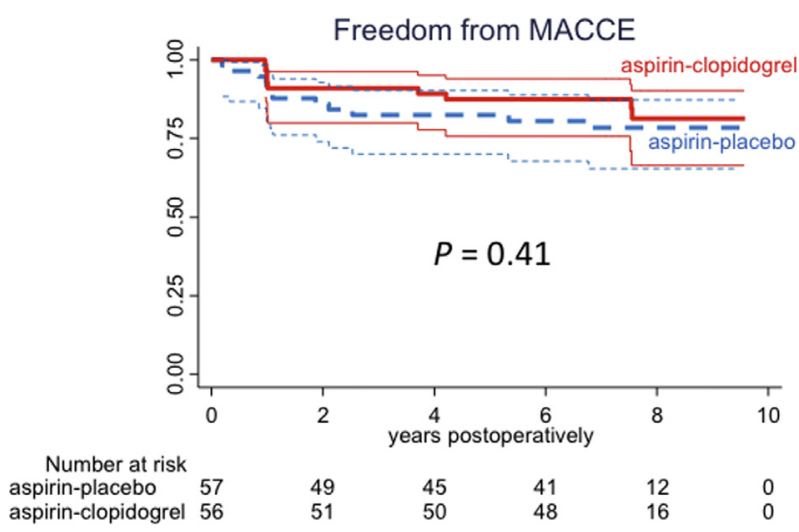

FIGURE 3. Freedom from major adverse cardiac or cerebrovascular events of the aspirin-clopidogrel group versus the aspirin-placebo group over the study period. Thick lines (full red lines for the aspirin-clopidogrel group and dashed blue lines for the aspirin-placebo group) depict the point estimates, whereas thin lines represent the $95 \%$ confidence limits. MACCE, Major adverse cardiac or cerebrovascular events.

We hypothesized that DAPT might be associated with less native CAD progression and improved SVG patency, resulting in better functional status. We found no significant difference in functional status or freedom from angina between the 2 groups, with or without DAPT. However, a trend toward improved long-term survival with freedom from MACCE/R, notably not driven by patients who had an acute coronary syndrome during the 6 weeks before original randomization, was observed in the group who took DAPT; that is, combining aspirin plus clopidogrel. It is possible that the absence of difference in functional status at 8 years may be explained by symptomatic patients having had a higher predisposition to MACCE/R or death, or even a higher rate of physiologic adaptation or acceptance of their symptoms. As a result, such confounders over time could

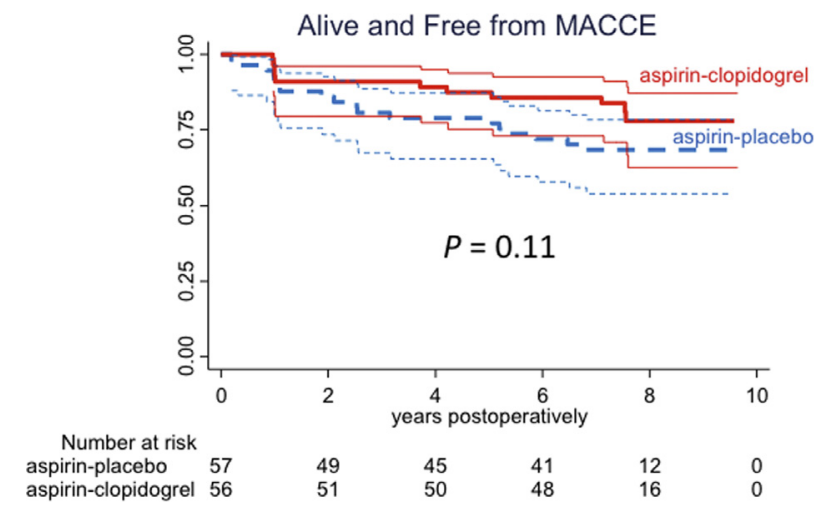

FIGURE 4. Patients alive and free from major adverse cardiac or cerebrovascular events in the aspirin-clopidogrel group versus the aspirin-placebo group over the study period. Thick lines (full red lines for the aspirin-clopidogrel group and dashed blue lines for the aspirin-placebo group) depict the point estimates, whereas thin lines represent the $95 \%$ confidence limits. MACCE, Major adverse cardiac or cerebrovascular events.
TABLE 3. Seattle Angina Questionnaire (SAQ) completion and responses to each subcomponent

\begin{tabular}{|c|c|c|c|}
\hline SAQ & Aspirin-clopidogrel & Aspirin-placebo & $\begin{array}{c}P \\
\text { value }\end{array}$ \\
\hline SAQ completion & $45 / 51(88.2 \%)$ & $41 / 48(85.4 \%)$ & .77 \\
\hline \multicolumn{4}{|c|}{ SAQ subcomponents responses } \\
\hline Physical limitation & $98.2 \pm 7.9$ & $97.7 \pm 7.5$ & .76 \\
\hline Angina stability & $91.1 \pm 19.7$ & $92.3 \pm 17.9$ & .78 \\
\hline Angina frequency & $97.4 \pm 11.8$ & $96.3 \pm 11.6$ & .67 \\
\hline Treatment satisfaction & $96.5 \pm 9.4$ & $95.3 \pm 10.1$ & .54 \\
\hline Disease perception & $94.5 \pm 9.1$ & $93.8 \pm 9.7$ & .73 \\
\hline
\end{tabular}

SAQ subcomponent responses are presented as mean \pm standard deviation. $S A Q$, Seattle Angina Questionnaire.

have reduced cross-sectional functional differences in surviving patients at 8 years post-CABG.

Although inconsistent with our previously published, shorter-term pooled analysis of DAPT after $\mathrm{CABG},{ }^{10}$ this trend toward improved MACCE-free long-term survival is intriguing and deserves further investigation. This trend toward improved MACCE-free long-term survival was not induced by the trend for increased use of bilateral internal thoracic arteries (BITA) in the aspirin-clopidogrel group. In fact, Table E1, which illustrates the characteristics of patients with MACCE/R, shows that treatment groups did not have a significant difference in the use of BITA $(18.2 \%$ in the aspirin-clopidogrel group and $10.5 \%$ in the aspirin-placebo group; $P=.61)$. Similarly, Table E2, which illustrates the characteristics of patients without MACCE/R, shows that treatment groups did not have a significant difference in the use of BITA $(26.7 \%$ in the aspirin-clopidogrel group and $15.8 \%$ in the aspirin-placebo group; $P=.29$ ). The trend for increased use of BITA in the aspirin-clopidogrel group was not statistically significant, but might have contributed to the trend in increased survival and reduced MACCE/R in this group. This being said, a recent randomized study, the Arterial Revascularization Trial (ART) trial, showed no

TABLE 4. Characteristics of grafts evaluated by computerized tomographic angiogram

\begin{tabular}{lccr}
\hline \multicolumn{1}{c}{ Grafts } & $\begin{array}{c}\text { Aspirin- } \\
\text { clopidogrel }\end{array}$ & $\begin{array}{c}\text { Aspirin- } \\
\text { placebo }\end{array}$ & $\begin{array}{c}\boldsymbol{P} \\
\text { value }\end{array}$ \\
\hline Total grafts $(\mathrm{N}=233)^{*}$ & 149 & 84 & \\
Total patent grafts $(\mathrm{n}=211)$ & $134(89.9 \%)$ & $77(91.7 \%)$ & .82 \\
\hline Total ITA $(\mathrm{n}=73)$ & 47 & 26 & 1.00 \\
\hline Patent ITA $(\mathrm{n}=67)$ & $43(91.5 \%)$ & $24(92.3 \%)$ & 1.00 \\
\hline Occluded ITA $(\mathrm{n}=6)$ & $4(8.5 \%)$ & $2(7.7 \%)$ & 1.00 \\
Total SVG $(\mathrm{n}=158)$ & 101 & 57 & 1.00 \\
\hline Patent SVG $(\mathrm{n}=142)$ & $90(89.1 \%)$ & $52(91.2 \%)$ & .79 \\
\hline Occluded SVG $(\mathrm{n}=16)$ & $11(10.9 \%)$ & $5(8.8 \%)$ & .79 \\
\hline
\end{tabular}

Values are presented as $\mathrm{n}$ or $\mathrm{n}(\%)$. ITA, Internal thoracic artery graft; $S V G$, saphenous vein graft. *Two grafts were radial artery grafts, which remained patent throughout the study. 
TABLE 5. Distribution of native coronary artery disease progression for each treatment group

\begin{tabular}{lccc}
\hline $\begin{array}{c}\text { Native coronary artery } \\
\text { disease }\end{array}$ & $\begin{array}{c}\text { Aspirin- } \\
\text { clopidogrel }\end{array}$ & $\begin{array}{c}\text { Aspirin- } \\
\text { placebo }\end{array}$ & $\begin{array}{c}\boldsymbol{P} \\
\text { value }\end{array}$ \\
\hline $\begin{array}{l}\text { Total native coronary } \\
\text { disease segments }(\mathrm{N}=200)\end{array}$ & 122 & 78 & \\
Grade of progression & & & \\
0 & $79(64.8)$ & $45(57.7)$ & .37 \\
1 & $36(29.5)$ & $20(25.6)$ & .63 \\
2 & $4(3.3)$ & $8(10.3)$ & .06 \\
3 & $1(0.8)$ & $2(2.6)$ & .56 \\
4 & $2(1.6)$ & $3(3.8)$ & .38 \\
\hline Values are presented as n $(\%)$. & & &
\end{tabular}

difference in MACCE/R or survival at 5 years when comparing BITA to single ITA. ${ }^{11}$ Despite some limitations (eg, significant crossover rate, high percentage of patients receiving a second arterial graft in the single ITA group and relatively short follow-up of 5 years), that study suggests that the potential benefits of BITA are expected to be observed after a longer period of time, and provides perspective on the limited role that BITA may have played in our cohort of patients over an only slightly longer followup. ${ }^{11}$

As for the other factors that may have contributed to the trend toward improved MACCE-free long-term survival, Table E2 shows that the patients without MACCE/R in the aspirin-clopidogrel group had a trend toward a lower incidence of hypertension $(66.7 \%$ for patients without MACCE/R in the aspirin-clopidogrel group, $84.2 \%$ for patients without $\mathrm{MACCE} / \mathrm{R}$ in the aspirin-placebo group; $P=.08$ ). This trend may have contributed to the trend in improved MACCE/R outcomes in the aspirin-clopidogrel group.

A previous analysis from the CASCADE randomized trial demonstrated that the combination of aspirin plus clopidogrel was associated with a slowing of the progression of native $\mathrm{CAD}$ after $\mathrm{CABG}{ }^{7}$ Our results again show a lower incidence of moderate to severe disease progression from preoperatively in the aspirin-clopidogrel group, rather than a difference in graft patency.

The rate of DAPT in the aspirin-placebo group after the 1year intervention duration of the trial was higher $(19.5 \%$ vs $10.9 \%$ ) than in the aspirin-clopidogrel group (Table 1). This difference, although intriguing and representing a reversal of the group's initial treatment allocation, was a result of patients being subjected to routine medical practice, involved a small minority of patients $(\sim 15 \%)$, was not statistically significant $(P=.37)$, and had limited effect on the difference in the progression of native CAD that was seen between both treatment groups. In fact, a lower incidence of moderate to severe disease progression was demonstrated in the aspirin-clopidogrel group when comparing the preoperative angiogram to the 8-year CCTA, and there was no difference in the incidence of more than mild native $\mathrm{CAD}$ progression between the 1-year angiogram and the 8-year CCTA. This implies that the DAPT had its greatest influence on the progression of native CAD during the first year of the study, and had limited influence from years 1 to 8 for this end point because there was no difference during that time period. Also, it is debatable as to which period of DAPT after bypass surgery or percutaneous coronary intervention may be more efficacious. In this regard, most studies so far have focused on the efficacy of DAPT during the first year after intervention, both in interventional cardiology and in cardiac surgery. ${ }^{8,10,12,13}$ Very few subsequent, long-term data exist, to which we believe that our study contributes significantly.

Table E3 of baseline characteristics in those who underwent CCTA shows that treatment groups did not have a significant difference in the use of left ITA $(100 \%$ in the aspirin-clopidogrel group and $100 \%$ in the aspirin-placebo group; $P=1.00)$, in the use of right ITA $(28.9 \%$ in the aspirin-clopidogrel group and $13.0 \%$ in the aspirinplacebo group; $P=.21)$, or in the use of BITA $(28.9 \%$ in the aspirin-clopidogrel group and $13.0 \%$ in the aspirinplacebo group; $P=.21$ ). The patency of those arterial grafts was similar between both treatment groups (Table 4) with $91.5 \%$ patency in the aspirin-clopidogrel group and $92.3 \%$ patency in the aspirin-clopidogrel group $(P=1.00)$. However, the nonsignificant trend for increased use of BITA in the aspirin-clopidogrel group might have contributed to the lower incidence of moderate to severe native disease progression, the increased survival, and the reduced MACCE/R that were observed in the aspirin-clopidogrel group. As mentioned previously, the use of BITA may have only played a limited role in our cohort of patients. ${ }^{11}$

This study represents a potentially important, first-time trend finding, which is hypothesis generating and deserves more investigation, especially because the majority of patients included in the CASCADE study did not have an acute coronary syndrome preoperatively.

The DAPT regimen that was used in the initial CASCADE trial combined aspirin plus clopidogrel. This regimen is currently considered as a low-intensity DAPT and is surpassed by higher-intensity DAPT combining aspirin and a more pronounced P2Y12 antagonist such as prasugrel or ticagrelor. ${ }^{12,13}$ Also, the initial dosage of clopidogrel that was used in the initial CASCADE trial (75 mg clopidogrel daily) may be considered low. ${ }^{6}$ Converting to a higher-intensity DAPT or to a higher dose of clopidogrel at inception might have enhanced the trends observed in this follow-up study; however, this would have had to be balanced with a higher risk of bleeding. These issues may justify a subsequent study, particularly with ticagrelor, which does not present the same partial response issues as clopidogrel. Presently, our clinical trial, named Ticagrelor Antiplatelet Therapy to Reduce Graft Events and Thrombosis, is investigating the effect of ticagrelor on vein graft patency as opposed to the 
current standard of care with the use of aspirin (ClinicalTrials.gov identifier: NCT02053909).

Although our long-term results showed no significant difference in graft patency between both groups, it clearly demonstrates a very high patency for SVG 8 years after CABG. Previous studies conducted almost 20 years ago indicated that only $60 \%$ of SVGs are patent at 10 years after $\mathrm{CABG}$, and of those that are patent, half have clinically significant stenoses. ${ }^{2,5}$ One possible explanation for the high patency of SVG in our follow-up study is the improvement in secondary prevention and our emphasis on post-CABG guidelines. ${ }^{14}$ During the past 20 years, the recommendations for secondary prevention after CABG have greatly changed and currently emphasize strict control of cardiovascular risk factors such as hypertension and low- and high-density lipoprotein levels. ${ }^{15,16}$

Compared with data from the initial CASCADE trial, ${ }^{6}$ we observed a higher rate of hypertension and diabetes mellitus over time. For instance, in the initial study, the rate of hypertension was $48.2 \%$ in the aspirin-clopidogrel group and $52.6 \%$ in the aspirin-placebo group. ${ }^{6}$ Although there is no significant difference between the use of antihypertension medications between both groups in this study (angiotensin-converting enzyme inhibitor: $43.5 \%$ in the aspirin-clopidogrel group and $48.8 \%$ in the aspirin-placebo group; $P=.67$ ), there was a significant difference in the rate of hypertension between both groups of study, with $64.7 \%$ in the aspirin-clopidogrel group and $83.3 \%$ in the aspirin-placebo group $(P=.03)$.

In this study, we found that patients had a very high adherence to long-term secondary prevention initiated around the time of their CABG. This was represented by their high compliance to the lipid-lowering medication, with $91.3 \%$ in the aspirin-clopidogrel group and $97.6 \%$ in the aspirinplacebo group $(P=.36)$, which is much higher than previously published data such as the Synergy Between Percutaneous Coronary Intervention With Taxus and Cardiac Surgery (SYNTAX) trial, where the use of a statin (for lipid-lowering) was only $75.5 \%$ after CABG. ${ }^{17}$ This high compliance was similarly observed for the long-term use of aspirin with an adherence of $87.0 \%$ in the aspirinclopidogrel group and $87.8 \%$ in the aspirin-placebo group $(P=1.0)$.

\section{Study Limitations}

This was not an a priori planned long-term analysis of the CASCADE trial. With only 113 patients at inception, complete late evaluation was achieved in a relatively small number of patients, making statistical inference challenging. Therefore, the findings of this study should be considered hypothesis generating. As with other smaller studies, the other limitation of our study is the issue of power to detect and claim differences, and we are underpowered to claim no significant difference between DAPT over aspirin. The strength

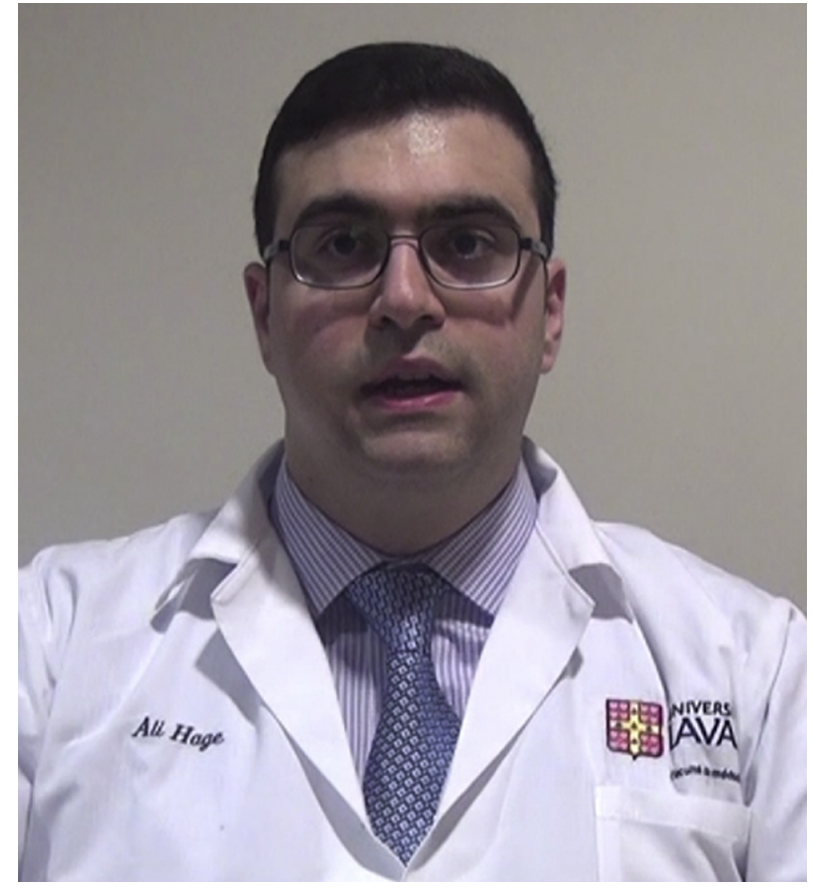

VIDEO 1. The primary author explaining the study and the importance of the article. Video available at: http://www.jtcvsonline.org/article/S00225223(17)31358-2/fulltext.

of the study is its uniqueness, very long duration of followup, and the added inferential power that may derive from it.

The evaluation of native CAD progression was based on the comparison of late follow-up results obtained by CCTA in relation to preoperative and 1-year conventional angiographic data. This has inherent pitfalls: the diagnostic accuracy and stenosis assessment of native arteries can also be limited in patients undergoing $\mathrm{CABG}$ due to severe coronary calcification, diffuse atherosclerosis, and small native disease. Despite these limitations, centers have reported very good accuracy in both grafts and native coronary arteries with CCTA. ${ }^{18-20}$ Weustink and colleagues $^{18}$ reported sensitivity, specificity, positive predictive value, and negative predictive value of $100 \%$, $96 \%, 97 \%$, and $100 \%$, respectively, for grafted native coronary arteries and $97 \%, 92 \%, 83 \%$, and $99 \%$ for native nongrafted coronaries. Initial assumptions of randomization may have been lost and bias may have been introduced, even if data presented in Tables E1-E3 reveal the late study groups not to be significantly different. In this regard, the modest size of the groups both may have made leveraging differences due to chance more likely to have occurred and less likely to reach statistical significance.

Although patients who completed the CCTA had similar characteristics between both treatment groups, there was an imbalance in follow-up between the aspirin-clopidogrel group and the aspirin-placebo group, reflected by the difference in the number of patients who completed the 
CCTA in each group. This may have also resulted from minor imbalances of randomization at baseline (eg, age, hypertension, and diabetes) that amplified over time. Also, some patients were made aware of the antiplatelet regimen allocation at the conclusion of the initial study because there was no rule against it once the initial CASCADE study was finished. Therefore, patients may have had different perceptions or levels of engagement.

Because this study was a cross-sectional follow-up, patients only reported their medication history at the time of the follow-up, and no information was obtained regarding any medication that had been taken in the interim. Further, the DAPT intervention was for 1 year, and afterward patients were left to receive best standard of care-which to this day remains unknown regarding the use of DAPT beyond 1 year in CABG patients. Although some numerical differences existed in medication use after the 1-year study intervention period was completed, none of these were dictated by the design of the study, and none reached statistical significance.

During the evaluation of graft patency, the degree of stenosis was initially reported using the FitzGibbon patency grading with grades A, B, or O. However, this grading was found to be less accurate with CCTA, and we resorted to a simpler, patent or not dichotomous grading system. ${ }^{21}$

Finally, there are discrepancies between individuals with regard to their response to clopidogrel. In fact, some studies report about $20 \%$ of nonresponders or poor responders to clopidogrel. ${ }^{22}$ Also, no aggregometry or genotyping tests to identify poor responders were performed in our study to identify poor responders.

\section{CONCLUSIONS}

At 8 years' follow-up, the addition of clopidogrel to aspirin during the first year after CABG did not significantly correlate with improved functional status or graft patency.

However, with a very high graft patency and freedom from death and MACCE/R, paralleled by high levels of adherence to secondary prevention measures after CABG, this long-term clinical trial cohort demonstrated a trend toward higher freedom from MACCE/R or death and a lower incidence of moderate to severe progression of native $\mathrm{CAD}$ in the aspirin-clopidogrel group, despite a lack of difference in graft patency. With the current lack of clear guidelines about the use of DAPT after CABG, this long-term follow-up study opens the door for new investigations to better understand and control the processes of native CAD progression and SVG disease after coronary artery bypass surgery (Video 1 ).

\section{Webcast}

You can watch a Webcast of this AATS meeting presentation by going to: http://webcast.aats.org/2016/ Video/Tuesday/05-17-16_Hall_E_1719_Hage-800.mp4.

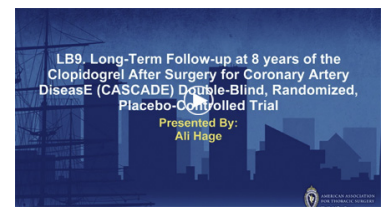

\section{Conflict of Interest Statement}

Authors have nothing to disclose with regard to commercial support.

\section{References}

1. Alderman EL, Kip KE, Whitlow PL, Bashore T, Fortin D, Bourassa MG, et al Bypass Angioplasty Revascularization Investigation. Native coronary disease progression exceeds failed revascularization as cause of angina after five years in the Bypass Angioplasty Revascularization Investigation (BARI). J Am Coll Cardiol. 2004;44:766-74.

2. Motwani JG, Topol EJ. Aortocoronary saphenous vein graft disease: pathogenesis, predisposition, and prevention. Circulation. 1998;97:916-31.

3. Herbert JM, Dol F, Bernat A, Falotico R, Lale A, Savi P. The antiaggregating and antithrombotic activity of clopidogrel is potentiated by aspirin in several experimental models in the rabbit. Thromb Haemost. 1998;80:512-8.

4. Clowes AW, Karnovsky MJ. Failure of certain antiplatelet drugs to affect myointimal thickening following arterial endothelial injury in the rat. Lab Invest. 1977; 36:452-64.

5. Fitzgibbon GM, Kafka HP, Leach AJ, Keon WJ, Hooper GD, Burton JR. Coronary bypass graft fate and patient outcome: angiographic follow-up of 5,065 grafts related to survival and reoperation in 1,388 patients during 25 years. J Am Coll Cardiol. 1996;28:616-26.

6. Kulik A, Le May MR, Voisine P, Tardif JC, Delarochelliere R, Naidoo S, et al. Aspirin plus clopidogrel versus aspirin alone after coronary artery bypass grafting: the clopidogrel after surgery for coronary artery disease (CASCADE) trial. Circulation. 2010;122:2680-7.

7. Une D, Al-Atassi T, Kulik A, Voisine P, Le May M, Ruel M. Impact of clopidogrel plus aspirin versus aspirin alone on the progression of native coronary artery disease after bypass surgery analysis from the Clopidogrel after Surgery for Coronary Artery Disease (CASCADE) randomized trial. Circulation. 2014;130(11 Suppl 1):S12-8.

8. Mauri L, Kereiakes DJ, Yeh RW, Driscoll-Shempp P, Cutlip DE, Steg PG, et al. Twelve or 30 months of dual antiplatelet therapy after drug-eluting stents. $N$ Engl J Med. 2014;371:2155-66.

9. Gaudino M, Tondi P, Benedetto U, Milazzo V, Flore R, Glieca F, et al. Radial artery as a coronary artery bypass conduit: 20-year results. J Am Coll Cardiol. 2016;68:603-10.

10. Verma S, Goodman SG, Mehta SR, Latter DA, Ruel M, Gupta M, et al. Should dual antiplatelet therapy be used in patients following coronary artery bypass surgery? A meta-analysis of randomized controlled trials. BMC Surg. 2015;15:1.

11. Taggart DP, Altman DG, Gray AM, Lees B, Gerry S, Benedetto U, et al. Randomized trial of bilateral versus single internal-thoracic-artery grafts. $N$ Engl J Med. 2016;375:2540-9.

12. Wiviott SD, Braunwald E, McCabe CH, Montalescot G, Ruzyllo W, Gottlieb S, et al. Prasugrel versus clopidogrel in patients with acute coronary syndromes. $N$ Engl J Med. 2007;357:2001-15.

13. Wallentin L, Becker RC, Budaj A, Cannon CP, Emanuelsson H, Held C, et al. Ticagrelor versus clopidogrel in patients with acute coronary syndromes. $N$ Engl J Med. 2009;361:1045-57.

14. Kulik A, Ruel M, Jneid H, Ferguson TB, Hiratzka LF, Ikonomidis JS, et al. Secondary prevention after coronary artery bypass graft surgery a Scientific Statement from the American Heart Association. Circulation. 2015;131.10:927-64.

15. Kulik A, Voisine P, Mathieu P, Masters RG, Mesana TG, Le May MR et al. Statin therapy and saphenous vein graft disease after coronary bypass surgery: analysis from the CASCADE randomized trial. Ann Thorac Surg. 2011;92:1284-91.

16. Jerzewski K, Ruel M, Voisine P, Le May MR, Kulik A. Does high-density lipoprotein influence the development of saphenous vein graft disease after coronary bypass surgery?: exploratory analysis from the CASCADE trial. J Cardiothorac Surg. 2013;8:172. 
17. Serruys PW, Morice MC, Kappetein AP, Colombo A, Holmes DR, Mack MJ, et al. Percutaneous coronary intervention versus coronary artery bypass grafting for severe coronary artery disease. N Engl J Med. 2009;360:961-72.

18. Weustink AC, Nieman K, Pugliese F, Mollet NR, Meijboom BW, van Mieghem C, et al. Diagnostic accuracy of computed tomography angiography in patients after bypass grafting: comparison with invasive coronary angiography. JACC Cardiovasc Imaging. 2009;2:816-24.

19. Ropers D, Pohle FK, Kuettner A, Pflederer T, Anders K, Daniel WG, et al. Diagnostic accuracy of noninvasive coronary angiography in patients after bypass surgery using 64-slice spiral computed tomography with 330-ms gantry rotation. Circulation. 2006;114:2334-41.

20. de Graaf FR, van Velzen JE, Witkowska AJ, Schuijf JD, van der Bijl N, Kroft LJ, et al. Diagnostic performance of 320-slice multidetector computed tomography coronary angiography in patients after coronary artery bypass grafting. Eur Radiol. 2011;21:2285-96.

21. Goldman S, Zadina K, Moritz T, Ovitt T, Sethi G, Copeland JG, et al. Long-term patency of saphenous vein and left internal mammary artery grafts after coronary artery bypass surgery: results from a Department of Veterans Affairs Cooperative Study. J Am Coll Cardiol. 2004:44:2149-56.

22. Campo G, Fileti L, Valgimigli M, Tebaldi M, Cangiano E, Cavazza C, et al. Poor response to clopidogrel: current and future options for its management. J Thromb Thrombolysis. 2010;30:319-31.

Key Words: antiplatelet drugs, coronary disease, coronary artery bypass graft

\section{Discussion}

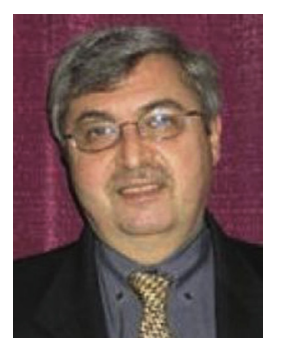

Dr Sary F. Aranki (Boston, Mass). I just want to quickly summarize how I interpret this study. The randomization occurred 8 years ago and was stopped after 1 year. So nobody was taking the medication after 1 year according to the protocol. It was left to the patients and their physicians. In fact, at the time of the follow-up at 8 years, there were more patients in the placebo group-10\% more-taking aspirin and clopidogrel than in the treatment group. There were more patients who were diabetic, who had a lot of hypertension compared with the placebo group, and also were taking proton pump inhibitors that can interfere with the response to clopidogrel. All these factors can cause progression of native coronary artery disease as well as graft disease.

So my first question to you is based on the way I interpret the study. Because the patients were no longer blinded, is this truly a blinded study? Is it truly randomized since they stopped taking the medication 7 years earlier? And is it really a placebo because they are not taking a placebo? I just want to understand if this is truly what you say in the title.

Dr Hage. Thank you very much for this question. As you indicated, patients were randomized, double-blinded and placebo during the first year of the trial. After the first year, the patients were aware of the regimen that they were put on. As we can see from the patient characteristics, there was not a significant difference between aspirin and clopidogrel between both groups.

Dr Aranki. My second question: You used follow-up computed tomography angiography to compare preoperative and 1-year angiograms and you found out that you have an amazing vein graft patency rate of $90 \%$ at 8 years. The last large randomized study, PREVENT IV [Project of Ex-Vivo Vein Graft Engineering via Transfection IV], showed that $25 \%$ of grafts had serious stenosis or occlusion at 12 to 18 years. What is the secret for this excellent patency rate?

Dr Hage. Thank you very much for this question. As we all know, 20 years ago when Dr Motwani and Dr FitzGibbon studied the saphenous vein graft, they showed that at 10 years of follow-up only $60 \%$ of SVGs are patent, and of those that are patent, almost 50\% have clinically significant stenosis. Since 20 years ago, the postcoronary artery bypass grafting guidelines and recommendations have greatly changed, and now we understand the cardiovascular risk factors with better control of hypertension and low and high lipid protein levels.

We think that the high adherence to secondary prevention that we saw in our study could explain the high patency of the grafts.

Dr Aranki. My last question: There are discrepancies between individuals with response to clopidogrel. It is supported that poor response occurs in about $40 \%$ of patients in some studies. Have you performed any aggregometry or genotyping tests to identify poor responders who would not benefit from clopidogrel treatment?

Dr Hage. There are some discrepancies in the genetics of patients and with their response to clopidogrel. At this time of the study, we did not analyze these discrepancies.

Dr Aranki. Thank you very much. Excellent work. 
TABLE E1. Characteristics of patients with major adverse cardiac or cerebrovascular events, including revascularization

\begin{tabular}{|c|c|c|c|}
\hline Characteristic & Aspirin-clopidogrel $(n=11)$ & Aspirin-placebo $(n=19)$ & $P$ value \\
\hline Age (y) & $75.54 \pm 7.68$ & $78.09 \pm 6.71$ & .35 \\
\hline Diabetes mellitus (\%) & $18.2(2 / 11)$ & $47.4(9 / 19)$ & .14 \\
\hline Female $(\%)$ & $18.2(2 / 11)$ & $26.3(5 / 19)$ & 1.00 \\
\hline $\begin{array}{l}\text { MI within } 6 \text { wk before } \\
\text { randomization }(\%)\end{array}$ & $36.4(4 / 11)$ & $15.8(3 / 19)$ & .37 \\
\hline Use of LITA graft $(\%)$ & $100(11 / 11)$ & $100(19 / 19)$ & 1.00 \\
\hline Use of RITA graft (\%) & $18.2(2 / 11)$ & $10.5(2 / 19)$ & .61 \\
\hline $\begin{array}{l}\text { Use of bilateral ITA grafts } \\
(\%)\end{array}$ & $18.2(2 / 11)$ & $10.5(2 / 19)$ & .61 \\
\hline Hypertension $(\%)$ & $90.9(10 / 11)$ & $84.2(16 / 19)$ & 1.00 \\
\hline Hypercholesterolemia (\%) & $90.9(10 / 11)$ & $78.9(15 / 19)$ & .63 \\
\hline Smoker $(\%)$ & $72.7(8 / 11)$ & $68.4(13 / 19)$ & 1.00 \\
\hline COPD $(\%)$ & $9.1(1 / 11)$ & $10.5(2 / 19)$ & 1.00 \\
\hline \multicolumn{4}{|c|}{ Medication use at the time of follow-up } \\
\hline ACE inhibitor $(\%)$ & $80.0(4 / 5)$ & $30.0(3 / 10)$ & .12 \\
\hline Beta blocker $(\%)$ & $40.0(2 / 5)$ & $50.0(5 / 10)$ & 1.00 \\
\hline Lipid-lowering (\%) & $80.0(4 / 5)$ & $100(10 / 10)$ & .33 \\
\hline $\begin{array}{l}\text { Calcium channel blocker } \\
\quad(\%)\end{array}$ & $20.0(1 / 5)$ & $40.0(4 / 10)$ & .60 \\
\hline Aspirin $(\%)$ & $80.0(4 / 5)$ & $80.0(8 / 10)$ & 1.00 \\
\hline Clopidogrel (\%) & $20.0(1 / 5)$ & $60.0(6 / 10)$ & .28 \\
\hline Proton pump inhibitor $(\%)$ & $20.0(1 / 5)$ & $60.0(6 / 10)$ & .28 \\
\hline
\end{tabular}

MI, Myocardial infarction; LITA, left internal thoracic artery graft; RITA, right internal thoracic artery graft; ITA, internal thoracic artery; COPD, chronic obstructive pulmonary disease; $A C E$, angiotensin-converting enzyme.

TABLE E2. Characteristics of patients without major adverse cardiac or cerebrovascular events, including revascularization

\begin{tabular}{|c|c|c|c|}
\hline Characteristic & Aspirin-clopidogrel $(n=45)$ & Aspirin-placebo $(\mathbf{n}=\mathbf{3 8})$ & $P$ value \\
\hline Age (y) & $73.2 \pm 7.70$ & $76.74 \pm 7.99$ & .04 \\
\hline Diabetes mellitus (\%) & $26.7(12 / 45)$ & $28.9(11 / 38)$ & 1.00 \\
\hline Female $(\%)$ & $6.7(3 / 45)$ & $5.3(2 / 38)$ & 1.00 \\
\hline $\begin{array}{l}\text { MI within } 6 \text { wk before } \\
\text { randomization }(\%)\end{array}$ & $13.3(6 / 45)$ & $21.1(8 / 38)$ & .39 \\
\hline Use of LITA graft (\%) & $100(45 / 45)$ & $97.4(37 / 38)$ & .46 \\
\hline Use of RITA graft (\%) & $26.7(12 / 45)$ & $15.8(6 / 38)$ & .29 \\
\hline $\begin{array}{l}\text { Use of bilateral ITA grafts } \\
\quad(\%)\end{array}$ & $26.7(12 / 45)$ & $15.8(6 / 38)$ & .29 \\
\hline Hypertension $(\%)$ & $66.7(30 / 45)$ & $84.2(32 / 38)$ & .08 \\
\hline Hypercholesterolemia (\%) & $86.7(39 / 45)$ & $92.1(35 / 38)$ & .50 \\
\hline Smoker $(\%)$ & $73.3(33 / 45)$ & $76.3(29 / 38)$ & .80 \\
\hline COPD $(\%)$ & $6.7(3 / 45)$ & $0(0 / 38)$ & .25 \\
\hline \multicolumn{4}{|c|}{ Medication use at the time of follow-up } \\
\hline ACE inhibitor $(\%)$ & $39.0(16 / 41)$ & $54.8(17 / 31)$ & .23 \\
\hline Beta blocker $(\%)$ & $65.9(27 / 41)$ & $74.2(23 / 31)$ & .61 \\
\hline Lipid-lowering (\%) & $92.7(38 / 41)$ & $96.8(30 / 31)$ & .63 \\
\hline $\begin{array}{l}\text { Calcium channel blocker } \\
(\%)\end{array}$ & $22.0(9 / 41)$ & $32.3(10 / 31)$ & .42 \\
\hline Aspirin $(\%)$ & $87.8(36 / 41)$ & $90.3(28 / 31)$ & 1.00 \\
\hline Clopidogrel (\%) & $9.8(4 / 41)$ & $6.5(2 / 31)$ & .69 \\
\hline Proton pump inhibitor $(\%)$ & $22.0(9 / 41)$ & $35.5(11 / 31)$ & .29 \\
\hline
\end{tabular}

$M I$, Myocardial infarction; LITA, left internal thoracic artery graft; RITA, right internal thoracic artery graft; ITA, internal thoracic artery; COPD, chronic obstructive pulmonary disease; $A C E$, angiotensin-converting enzyme. 
TABLE E3. Characteristics of patients who underwent coronary computed tomographic angiography

\begin{tabular}{|c|c|c|c|}
\hline Characteristic & Aspirin-clopidogrel $(\mathbf{n}=\mathbf{3 8})$ & Aspirin-placebo $(\mathbf{n}=\mathbf{2 3})$ & $P$ value \\
\hline Age $(y)$ & $70.99 \pm 7.27$ & $74.44 \pm 7.25$ & .08 \\
\hline Diabetes mellitus (\%) & $23.7(9 / 38)$ & $34.8(8 / 23)$ & .39 \\
\hline Female $(\%)$ & $5.3(2 / 38)$ & $17.4(4 / 23)$ & .19 \\
\hline $\begin{array}{l}\text { MI within } 6 \text { wk before } \\
\text { randomization (\%) }\end{array}$ & $18.4(7 / 38)$ & $21.7(5 / 23)$ & .75 \\
\hline Use of LITA graft (\%) & $100(38 / 38)$ & $100(23 / 23)$ & 1.00 \\
\hline Use of RITA graft (\%) & $28.9(11 / 38)$ & $13.0(3 / 23)$ & .21 \\
\hline $\begin{array}{l}\text { Use of bilateral ITA grafts } \\
(\%)\end{array}$ & $28.9(11 / 38)$ & $13.0(3 / 23)$ & .21 \\
\hline Hypertension (\%) & $65.8(25 / 38)$ & $82.6(19 / 23)$ & .24 \\
\hline Hypercholesterolemia (\%) & $89.5(34 / 38)$ & $95.7(22 / 23)$ & .64 \\
\hline Smoker $(\%)$ & $76.3(29 / 38)$ & $78.3(18 / 23)$ & 1.00 \\
\hline COPD $(\%)$ & $5.3(2 / 38)$ & $0(0 / 23)$ & .52 \\
\hline \multicolumn{4}{|c|}{ Medication use at the time of follow-up } \\
\hline ACE inhibitor $(\%)$ & $42.1(16 / 38)$ & $65.2(15 / 23)$ & .11 \\
\hline Beta blocker $(\%)$ & $68.4(26 / 38)$ & $65.2(15 / 23)$ & 1.00 \\
\hline Lipid-lowering (\%) & $92.1(35 / 38)$ & $100(23 / 23)$ & .28 \\
\hline $\begin{array}{l}\text { Calcium channel blocker } \\
\quad(\%)\end{array}$ & $18.4(7 / 38)$ & $30.4(7 / 23)$ & .35 \\
\hline Aspirin $(\%)$ & $84.2(32 / 38)$ & $87.0(20 / 23)$ & 1.00 \\
\hline Clopidogrel (\%) & $13.2(5 / 38)$ & $17.4(4 / 23)$ & .72 \\
\hline Proton pump inhibitor $(\%)$ & $21.1(8 / 38)$ & $34.8(8 / 23)$ & .37 \\
\hline
\end{tabular}

MI, Myocardial infarction; LITA, left internal thoracic artery graft; RITA, right internal thoracic artery graft; ITA, internal thoracic artery; COPD, chronic obstructive pulmonary disease; $A C E$, angiotensin-converting enzyme. 